\title{
Modeling and optimizing the gas-lift effect system for texturing silicon
}

\author{
M Amouzgar, M Kahrizi \\ Electrical and Computer Engineering Department, Concordia University, 1515 St. \\ Catherine West, EV005.139 Montreal, Quebec, Canada H3G 2W1 \\ Email: m_amouzg@encs.concordia.ca
}

\begin{abstract}
Modeling and simulation techniques were used to understand the fluid flow patterns inside the silicon texturing system operating under gas-lift effect (GLE) for solar cell applications. Experiments were performed which confirmed the validity of the simulation model and the simulated results. It was determined that due to the original non-optimal inlet, the fluid flow pattern and the non-uniform fluid velocity distribution inside the system resulted in the generation of lower velocity regions on the surface of the textured silicon. The simulation tool validated the correlation of the lower fluid velocity with the reduced surface coverage, uniformity and subsequent less optimal surface reflectivity. Various inlet designs were modeled and evaluated for optimal performance. The best case inlet design was fabricated and tested resulting in the validation of the simulation work and significant improvement in the GLE texturing system performance. With the new inlet design, as the fluid velocity reaches and goes beyond some critical value (in this case $0.047 \mathrm{~m} / \mathrm{s}$ ) in areas that had shown lower velocity in the original inlet design (mainly close to the inlet and side walls), the observed morphology and surface reflectivity improve significantly with values very similar to those of the higher-velocity areas.
\end{abstract}

PACS 78.40.Fy, 88.40.hm

\section{Introduction}

Lowering the reflectivity of the surface of silicon-based solar cells is an on-going challenge. The anisotropic etching technique is one of the most cost-effective ways for texturing the silicon surface to achieve low reflectivity. However, the hydrogen bubbles generated during the process, lead to the creation of the gas blanketing effect on the surface of the silicon preventing a uniform surface texturing. The traditional methods for removing the bubbles involve adding surface active agents (surfactants) such as Isopropyl alcohol (IPA) [1] to modify the liquid surface tension resulting in the quicker release of the hydrogen bubbles from the silicon surface. However, there are unwanted effects as a result of adding IPA such as less control over the etching rate and the average particle size.

In previous work, we reported a new approach for the removal of the bubbles requiring only half the IPA amount by taking advantage of the gas-lift effect (GLE) [2]. As hydrogen bubbles become large enough, they detach from the silicon surface and rise to the surface of the liquid. The rising velocity of the bubbles reaches a terminal value which is a function of the bubble diameter as well as the liquid viscosity as defined by the Stokes law. As a result of this rising, the bubbles push the liquid upwards, and at the same time, induce a vacuum underneath leading to 
more liquid suction into the system causing a pumping effect and a natural circulation that stays in effect as long as the bubbles are generated, detach and rise to the surface inside the enclosed GLE texturing system.

Due to a higher liquid velocity inside the GLE system, the hydrogen bubbles attached to the silicon surface are removed much quicker. As a result, the silicon surface is more exposed to the TMAH etching solution leading to higher textured surface area.

However, due to non-uniform velocity distribution inside the GLE system caused by the nonoptimal geometry of the inlet, regions with non-uniform reflectivity are formed. We believe that a modification to the GLE structure will be instrumental to achieve uniform texturing of the silicon surface.

The present study focuses on the use of simulation tools for the optimization of the inlet design and the modification of the flow patterns during the silicon texturing based on the innovative GLE approach. Different inlet geometries were designed, modeled and studied. The best-case inlet design was fabricated and further experiments using the new inlet verified our simulation results confirming a significant reduction of lower velocity regions, and therefore, maximizing surface uniformity.

\section{Modeling and experimental details}

\subsection{Modeling tool and assumptions}

A 3D modeling of the GLE system was performed using COMSOL finite element analysis simulation software version 4.1. The following approximations were considered to simplify the modeling:

- The effect of micro agitation and turbulence created by the silicon surface mesh has been ignored.

- The silicon surface is assumed to be a polished flat surface parallel to the glass surface.

- The Gas-Lift effect due to the hydrogen bubble generation and rising has been modeled as a mechanical pump generating a flow out of the GLE system.

- The flow of the liquid inside the GLE system, based on the generation of the hydrogen bubbles and their gas-lift effect, was assumed to be constant in all cases. Then a liquid velocity at the outlet for each case was assumed to be the same and not dependent on the inlet geometry.

\subsubsection{GLE geometry and dimensions}

Table 1 lists the geometric parameters and their values for the modeled non-optimal GLE system. Inlets with various geometries were designed and modeled to identify the one with most uniformly distributed resistance resulting in the uniform fluid velocity distribution inside the GLE system.

Table 1 : Dimensions of the modeled non-optimal GLE system

\begin{tabular}{|l|l|l|}
\hline Parameter & Value & Unit \\
\hline GLE active area width & 25 & $\mathrm{~mm}$ \\
\hline GLE active area height & 50 & $\mathrm{~mm}$ \\
\hline GLE gap & 2 & $\mathrm{~mm}$ \\
\hline
\end{tabular}




\subsubsection{Turbulent flow modelling equations, constants and variables}

The flow was considered to be isothermal and in turbulent state. The standard k- $\varepsilon$ (k-epsilon) is classified as eddy viscosity model and is the most widely used [3] [4]. This two-equation model includes two extra transport equations to represent the turbulent properties of the flow [5][6][7]. Transport equations are solved for two scalar properties of turbulence. The k-equation is a model of the transport equation for the turbulent kinetic energy. The $\varepsilon$-equation is the model for the dissipation rate of turbulent kinetic energy [8].

One of the two main references on this model is described by Launder and Sharma [9]. The Reynolds stresses are modeled as follows:

$\tau_{t i j}=2 \mu_{t}\left(S_{i j}-S_{n n} \delta_{i j} / 3\right)-2 \rho k \delta_{i j} / 3$, where $\mu_{t}=c_{\mu} \rho k^{2} / \varepsilon$ is the eddy viscosity defined as a

function of the turbulent kinetic energy $(\mathrm{k})$ and the turbulent dissipation $(\varepsilon)$, $S_{i j}=\frac{1}{2}\left(\frac{\partial u_{i}}{\partial x_{j}}+\frac{\partial u_{j}}{\partial x_{i}}\right)$ is the mean-velocity strain-rate tensor, $\rho$ is the fluid density, $\mathrm{k}$ is the turbulent kinetic energy, and $\delta_{i j}$ is the Kronenecker delta.

Table 2 contains the turbulence transport equations for the k- $\varepsilon$ Launder-Sharma model.

Table 2: Equations used in the modeling of the GLE system by COMSOL

\begin{tabular}{|c|l|}
\hline \multicolumn{1}{|c|}{ Equation } & \multicolumn{1}{c|}{ Description } \\
\hline$\frac{\partial \rho k}{\partial t}+\frac{\partial}{\partial x_{j}}\left(\rho u_{j} \frac{\partial k}{\partial x_{j}}-\left(\mu+\frac{\mu_{\tau}}{\sigma_{k}}\right) \frac{\partial k}{\partial x_{j}}\right)=\tau_{t i j} S_{i j}-\rho \varepsilon$ & $\begin{array}{l}\text { Turbulence energy } \\
\text { transport }\end{array}$ \\
\hline$\frac{\partial \rho \varepsilon}{\partial t}+\frac{\partial}{\partial x_{j}}\left(\rho u_{j} \varepsilon-\left(\mu+\frac{\mu_{\tau}}{\sigma_{\varepsilon}}\right) \frac{\partial \varepsilon}{\partial x_{j}}\right)=c_{\varepsilon 1} \frac{\varepsilon}{k} \tau_{t i j} S_{i j}-c_{\varepsilon 2} \rho \frac{\varepsilon^{2}}{k}$ & $\begin{array}{l}\text { Energy dissipation } \\
\text { transport }\end{array}$ \\
\hline
\end{tabular}

The model constants are defined as follows:

$c_{\mu}=0.09, c_{\varepsilon 1}=1.45, c 2=1.92, \sigma_{k}=1.0, \sigma_{\varepsilon}=1.3$

Using the turbulence variables, $\mathrm{k}$ and $\varepsilon$, it is possible to account for history effect such as convection and diffusion of turbulent energy.

Boundary conditions at surfaces (walls) was assumed to be no-slip and were set to $\mathrm{k}=0$ and $\varepsilon=$ 0 . Zero-gradient conditions are applied at symmetry boundaries. Inlet boundary condition was set to zero pressure with no viscous stress, and for the outlet, the normal outflow velocity was assumed.

\subsection{Experimental measurement of the modeling parameters}

The fluid viscosity was measured using a Paar Phisica MCR 500 Rheometer. The density of the liquid was measured using a Micromeritics Accupyc II 1340 Pycnometer. The average velocity in the GLE system was calculated by measuring the time it took a red dye to travel the entire length of the GLE system $(50 \mathrm{~mm})$. A digital video camera and a digital counter were used to record the entering and exiting times of the red dye into and out of the GLE system. The experiments were repeated 10 times and an average value was obtained to minimize the error. The average velocity was used to calculate the flow rate out of the GLE system. 
The flow rate at the inlet was calculated by multiplying the inlet area by the measured average fluid velocity inside the system. For an empirically measured average velocity of $0.05 \mathrm{~m} / \mathrm{s}$ and an inlet cross section area of $0.00005 \mathrm{~m}^{2}$ the flow at the inlet was calculated to be $0.0025 \mathrm{l} / \mathrm{s}$.

\subsubsection{Silicon Texturing Experimental Setup}

P-type (100) orientation silicon wafers with a thickness of $625+/-25 \mu \mathrm{m}$ and a resistivity of $10-20$ $\Omega$-cm were purchased from Silicon Inc. Tetramethylammonium hydroxide (TMAH) $25 \mathrm{Wt}$. \% was purchased from Sachem Inc. and diluted with deionized (DI) water to reach the desired 2 wt.\% concentrations used in the experiments. 99\% IPA was purchased from VWR International and was added to the etching solution to obtain weight concentrations of $3.5 \%$. The experimental setup for texturing using the GLE approach as well as the characterization and analysis tools are exactly the same used in our previous work [2]. The specular reflectivity was measured for all samples in our experiments and then converted to specular solar weighted average by integrating the product of the reflectance and the AM1.5 photon density, divided by the total number of photons between $300 \mathrm{~nm}$ and $800 \mathrm{~nm}$.

\section{Results and Discussions}

\subsection{Effect of inlet geometry on flow pattern during the silicon surface texturing}

The accumulation of hydrogen bubbles during the silicon texturing process results in a nonoptimal textured surface leading to a non-desirable higher reflectivity values [10] [11]. The conventional approach has been the use of significant amounts of IPA or other surfactants to facilitate the removal of hydrogen bubbles to obtain a textured surface with lowered reflectivity. In our previous paper [2], we reported the efficacy of the GLE effect on the removal of the hydrogen bubbles which allows obtaining the same desired lower level of reflectivity by using significantly lower amounts of IPA. However, owing to non-uniform flow pattern inside the used GLE system, regions with lower fluid velocity are formed inside the GLE system resulting in a different reflectivity values for those regions compared to the rest of the textured surface.

Figure 1 illustrates the presence of these regions mostly at the inlet and closer to both sidewalls of the system (areas surrounded by the dashed lines). The texturing parameters used for this sample were the same as the ones resulting in the best reflectivity case reported in our previous work [2]. Accordingly, the values of the temperature, GLE gap size as well as the concentrations of TMAH and IPA were, $90{ }^{\circ} \mathrm{C}, 2 \mathrm{~mm}, 2 \%$ and $3.5 \%$, respectively.

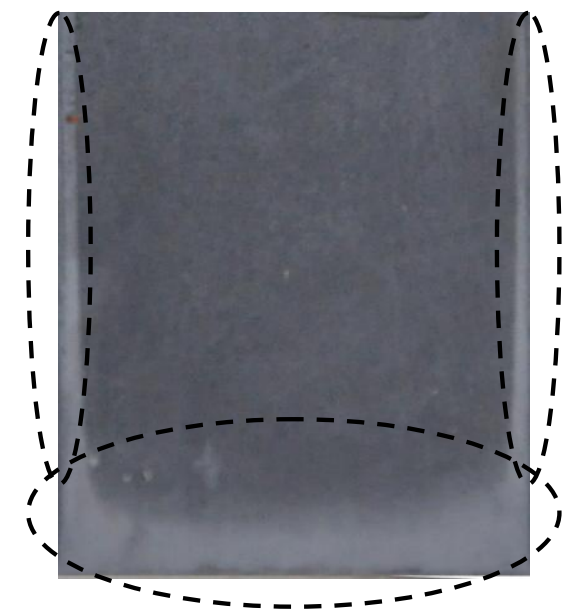

Figure 1: Low velocity areas formed by the GLE system 
The morphology and the reflectivity of these lower-velocity areas were compared to those of the rest of the surface using scanning electron microscope (SEM) analysis and spectrophotometry measurements.

Figure 2 illustrates the morphology of the textured surface of the silicon using the GLE system. As it maybe seen, the areas with higher velocity (figure 2a) show significantly higher textured surface coverage compared to the lower-velocity areas (figure 2b). Accordingly, the textured surface coverage values determined by SEM image analysis (expressed as the percentage of the surface covered by the formed pyramids with respect to the total surface area) were about $99 \%$ and $68 \%$ for higher and lower velocity areas, respectively. It may also be noted that the higher fluid velocity results in a more uniform pyramids size distribution.

The specular reflectance spectra of these two distinct areas presented in figure 3 , also confirm the lower reflectivity of the higher-velocity regions over the entire spectrum (400 to $800 \mathrm{~nm}$ ).
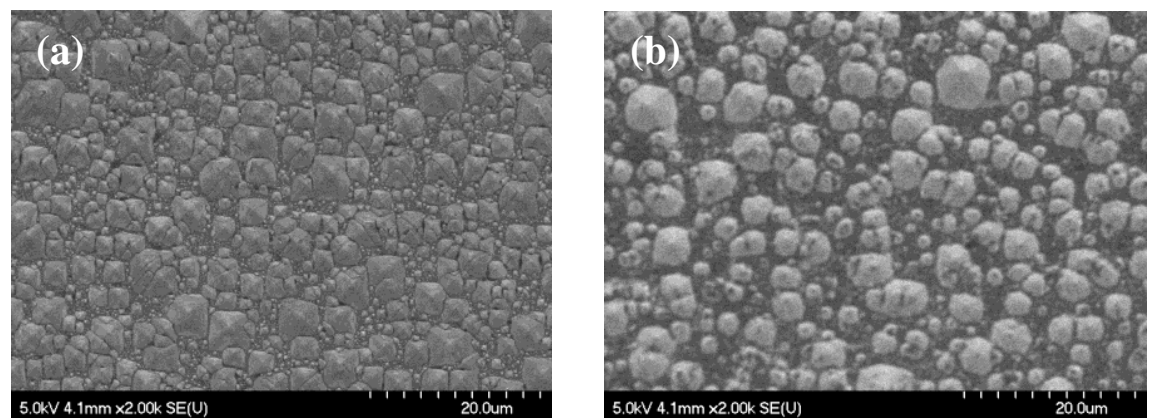

Figure 2: Surface of textured silicon in the (a) higher-velocity and (b) lower-velocity areas

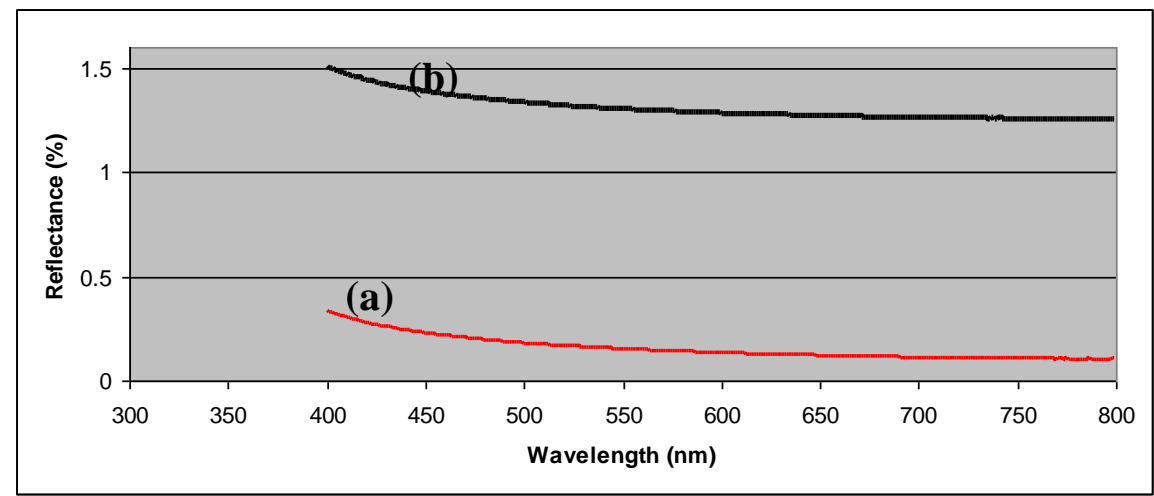

Figure 3: The reflectivity of the (a)higher-velocity areas vs. (b)lower-velocity areas

The areas affected by the fluid lower velocity (and consequently, higher reflectivity) represent about $15 \%$ of the overall surface, and it is important from an economical point of view, to minimize their surface if the GLE approach is to be used as a viable alternative to the conventional texturing techniques at an industrial scale.

As it was assumed that the formation of these lower-velocity areas was related to the geometry of the GLE system inlet, it was decided to use a simulation tool to model and validate this assumption as well as to improve the design of the inlet. 


\subsection{Modeling of the flow patterns}

\subsubsection{Velocity distribution of non-optimal inlet design}

COMSOL software application was used as the simulation tool to obtain a model that can closely represent the formation of the distinctive textured areas observed experimentally in the GLE system using the initial non-optimal inlet design. Table 3 summarizes the parameters and their values used for the modeling. The fluid density, viscosity and flow rate were measured under the same experimental conditions as those used for the texturing of the silicon surface sample presented in figure 1 (section 3.1).

Table 3: Parameters and their values used for the modeling

\begin{tabular}{|l|l|l|}
\hline Name & Value & Description \\
\hline$\rho$ & $1043\left[\mathrm{~kg} / \mathrm{m}^{3}\right]$ & Fluid density \\
\hline$\mu$ & $0.6 \mathrm{e}-3\left[\mathrm{~N}^{*} \mathrm{~s} / \mathrm{m}^{2}\right]$ & Fluid viscosity \\
\hline Flow Rate & $0.0025[1 / \mathrm{s}]$ & Electrolyte flux at the inlet \\
\hline $\mathrm{V}_{\mathrm{avg}}$ & $0.05[\mathrm{~m} / \mathrm{s}]$ & Average liquid velocity \\
\hline $\mathrm{G}$ & $9.81\left[\mathrm{~m} / \mathrm{s}^{2}\right]$ & Gravitational constant \\
\hline
\end{tabular}

Figure 4 illustrates the liquid velocity pattern inside the modelled GLE system.

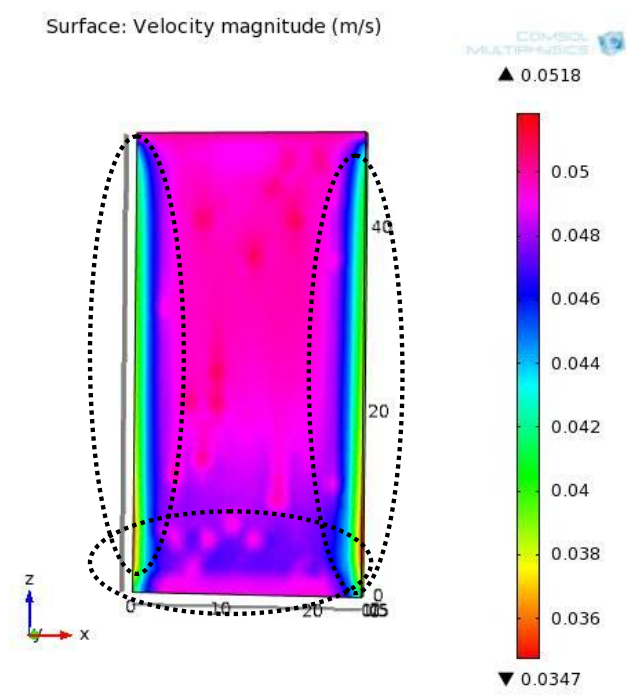

Figure 4 : The velocity pattern inside the GLE system

The surface areas designated by the dotted ovals identify the lower fluid velocity areas. As it may be noted, the model predicts the formation of lower velocity regions close to the inlet and the side walls as observed experimentally (see figure 1). The predicted velocity profile across the GLE system width ( $\mathrm{x}$ direction) for several heights ( $\mathrm{z}$ direction) on the silicon surface $(\mathrm{y}=0)$ are illustrated in figure 5. As it maybe seen, lower velocity regions represent up to $25 \%$ of the entire width of the GLE system and, in areas close to the sidewalls, the reduction in velocity can reach up to $30 \%$ compared to the high velocity regions. The model also predicts lower velocities in areas close to the inlet as it maybe noted from the velocity profile at $4 \mathrm{~mm}$ height, as also observed in the experimental sample. In addition, the model shows some fluctuations in the velocity value along the width of the GLE system regardless of the height. 


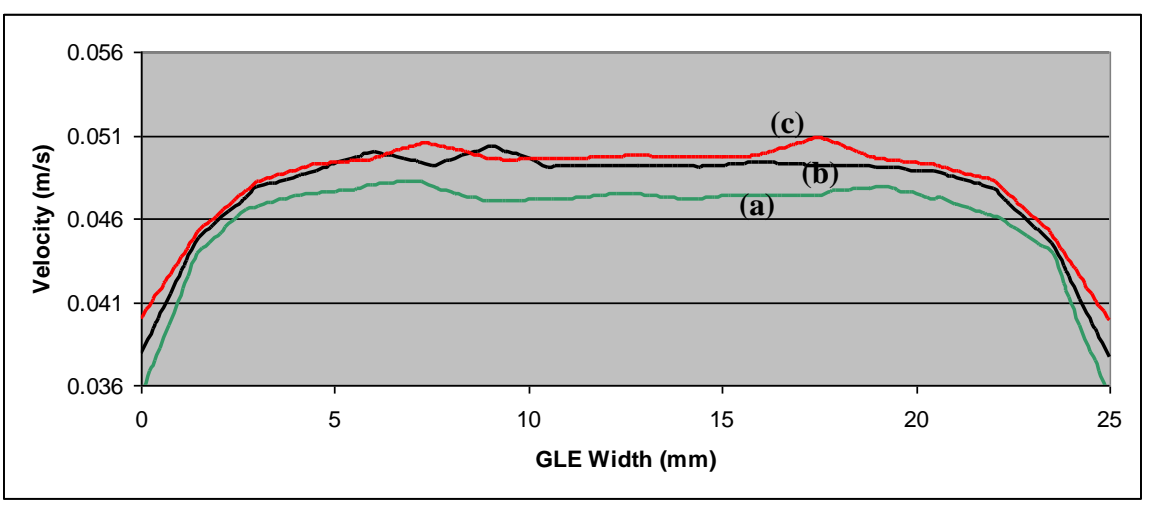

Figure 5 : Velocity magnitude (m/s) distribution in the GLE system at (a) $4 \mathrm{~mm}$, (b) $20 \mathrm{~mm}$, and (c) $40 \mathrm{~mm}$ height

\subsubsection{Optimization of the GLE inlet design}

It was decided to use a design that would result in a higher pressure at the inlet leading to a more uniform fluid flow rate and a better velocity distribution profile. Accordingly, two sets of inlet geometry configurations were designed and tested with the simulation tool to evaluate their influence on the flow pattern and any improvements on the lower-velocity regions. One consisted of an inlet with a slit gap, varying between 0.2 and $1.8 \mathrm{~mm}$ (increments of $0.1 \mathrm{~mm}$ ), all along the width of the GLE system. The other, was an array of small size holes $(0.5 \mathrm{~mm}$ in diameter) with a number of holes varying between 5 and 15 along the width of the inlet. The best case result was found to be the inlet design with a slit of $1.2 \mathrm{~mm}$ gap as illustrated in figure 6 .
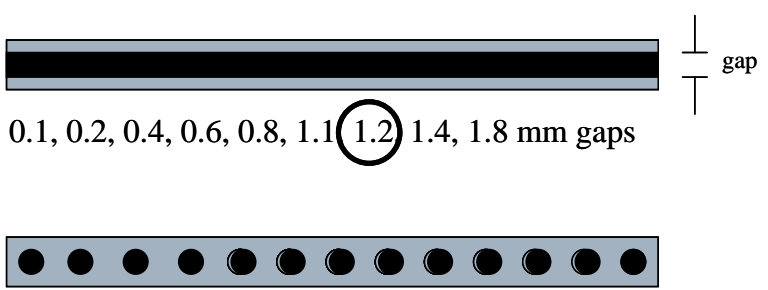

$0.1-0.55 \mathrm{~mm}$ circle radius

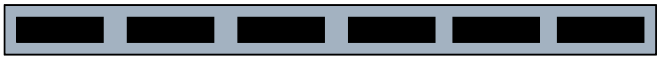

$0.1-1.8 \mathrm{~mm}$ wide rectangles

Figure 6: Designed and tested inlets using the simulation tool

Figure 7 illustrates the velocity distribution of the electrolyte inside the GLE system with such inlet design. The most noticeable result is the improvements in velocity in the two regions close to the side walls. However, the velocity improvements at the lower heights (closer to the inlet) are less significant and still some velocity fluctuations remain at these lower heights (4 mm height). 


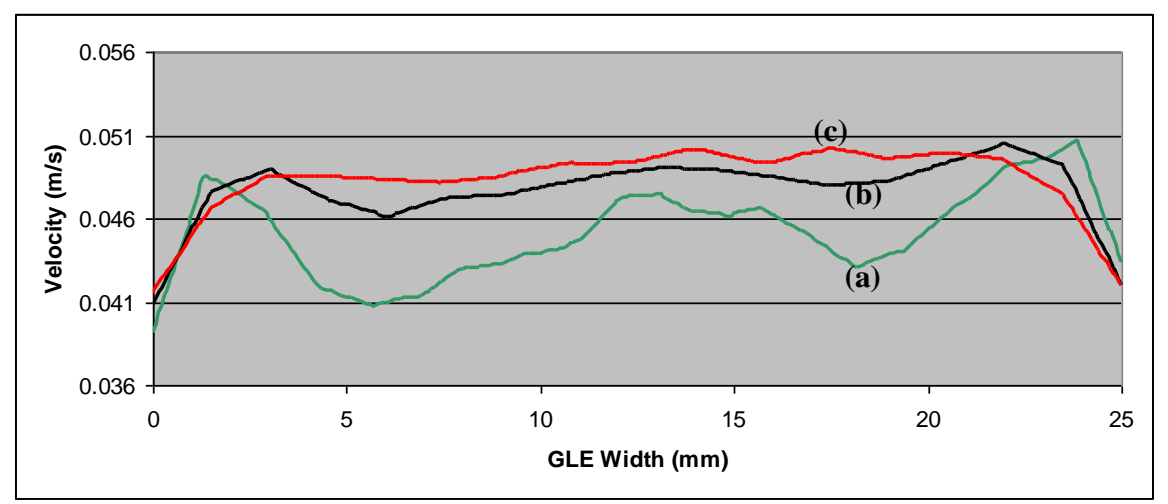

Figure 7: Velocity magnitude (m/s) distribution in the GLE system with $1.2 \mathrm{~mm}$ slit inlet design at (a) $4 \mathrm{~mm}$, (b) $20 \mathrm{~mm}$, and (c) $40 \mathrm{~mm}$ height

It was assumed that this effect may be attributed to the edge effect related to sharp corners of the rectangular slit. As a result, an improved version of the same slit was designed and tested with rounded corners with a radius of $0.55 \mathrm{~mm}$ as illustrated by figure 8 .

Figure 8: The best case inlet design with a $1.2 \mathrm{~mm}$ gap and rounded corners of $0.55 \mathrm{~mm}$ radius

The velocity distribution in the GLE system inlet design with the $1.2 \mathrm{~mm}$ gap and rounded corners is represented in figure 9. It maybe noticed that the latest inlet design addresses the three main issues that had been targeted to be resolved in the non-optimal design. First, the liquid velocities in the two extremities near the side walls have improved significantly. Secondly, the velocity at the very bottom of the GLE system height has also improved significantly. Finally, there are fewer liquid velocity fluctuations at all heights of the GLE system (4 mm, $20 \mathrm{~mm}$ and 40 $\mathrm{mm})$.

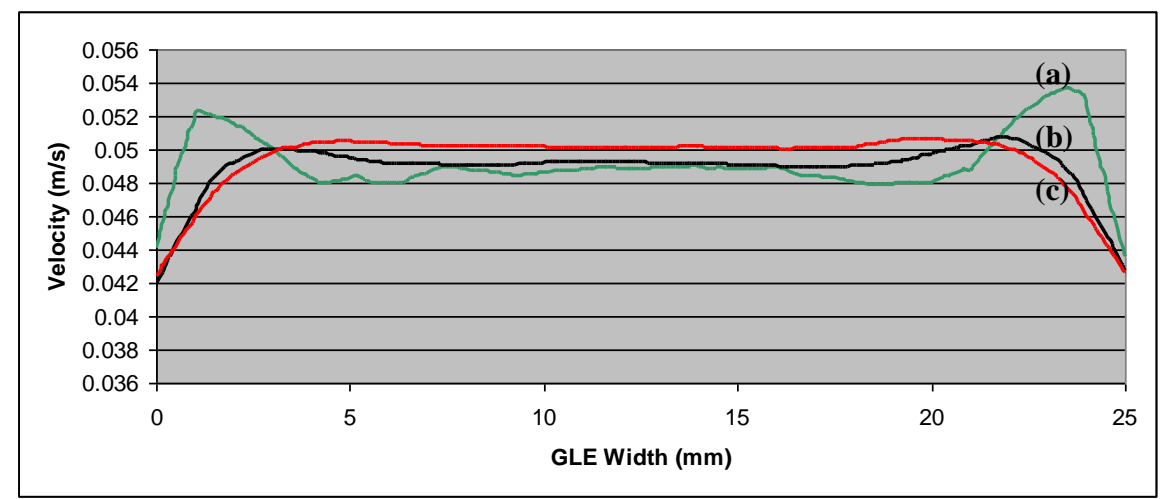

Figure 9: Velocity magnitude (m/s) distribution in the GLE system with a $1.2 \mathrm{~mm}$ gap slit inlet with rounded corners at (a) $4 \mathrm{~mm}$, (b) $20 \mathrm{~mm}$, and (c) $40 \mathrm{~mm}$ height

The improvements of the flow pattern of the new design can better be appreciated by comparing its velocity profile at low heights with that of the non-improved design as presented in figure 10. In fact, as it may be seen, while more than $45 \%$ of the width of the GLE system are at velocities 
below $0.047 \mathrm{~m} / \mathrm{s}$ in the case of the non-optimal inlet, less than $4 \%$ of the total width falls below this velocity for the improved inlet design.

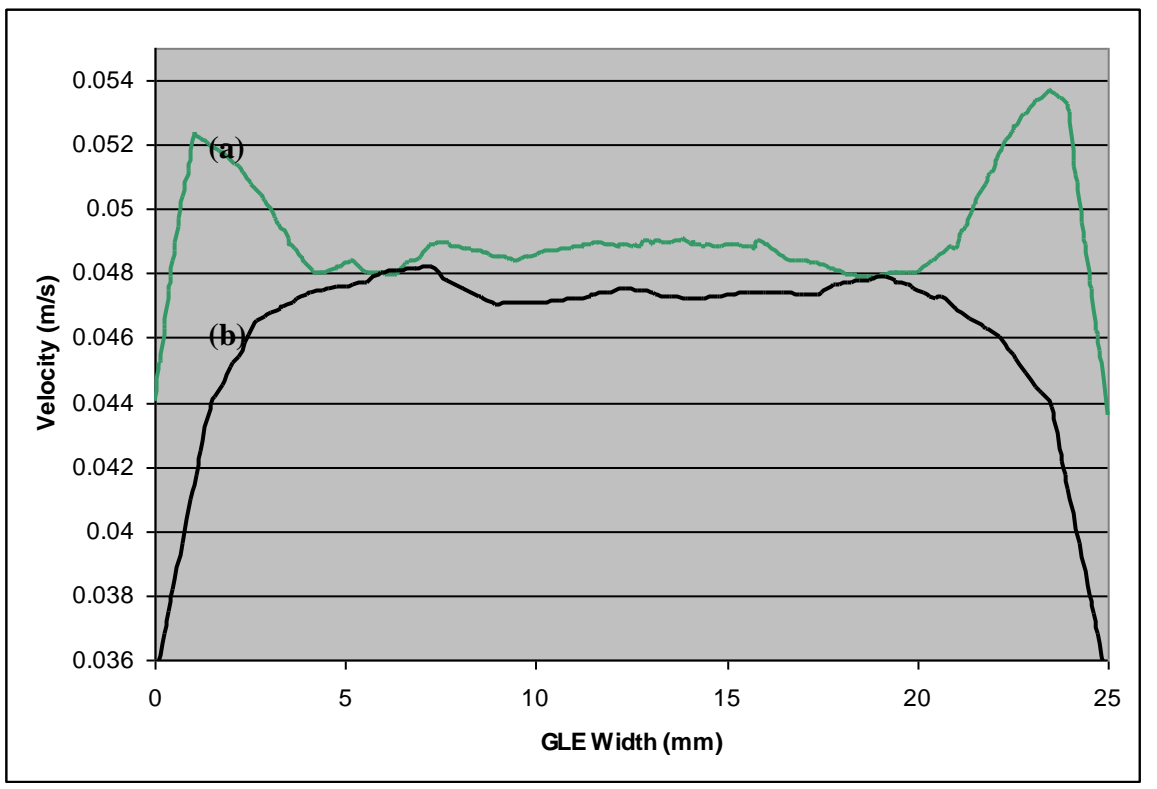

Figure 10: The velocity distribution at $4 \mathrm{~mm}$ height for the (a) $1.2 \mathrm{~mm}$ inlet with round corners versus the (b) $2 \mathrm{~mm}$ wide original non-optimal inlet

\subsection{Experimental results based on the new inlet design}

A new GLE system was fabricated based on the improved $1.2 \mathrm{~mm}$-gap inlet with rounded corners and used to perform silicon surface texturing under the same experimental conditions. Figure 11 illustrates the efficacy of the new inlet design in improving the uniformity of the textured sample silicon surface by significantly reducing the low velocity regions highlighted by the dashed ovals in figure $11 \mathrm{~b}$.
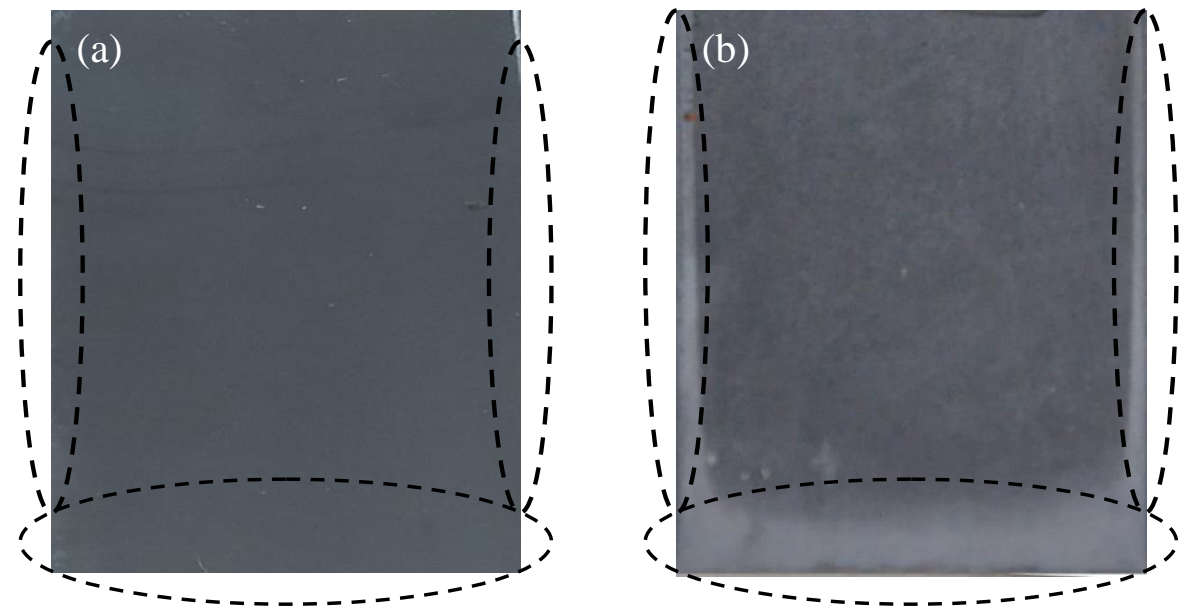

Figure 11: Silicon surfaced textured (a) with the optimized GLE system and (b) without 
When the two samples are visually compared, the lower-velocity areas close to the inlet and the side walls of the sample with the optimized inlet have practically disappeared, suggesting the improved velocity flow pattern as a consequence of the improved inlet design. This is also confirmed when the difference in morphology and the reflectance are examined for both inlet designs using SEM and spectrophotometry respectively. As it may be seen in figure 12, the regions close to the side walls and the inlet (indicated by the dashed ovals in figure 11a), present a morphology similar to the higher-velocity areas in the case of the improved inlet design. In fact the value of the surface coverage is practically the same (more than 99\%) compared to the nonoptimal design (about 68\%).
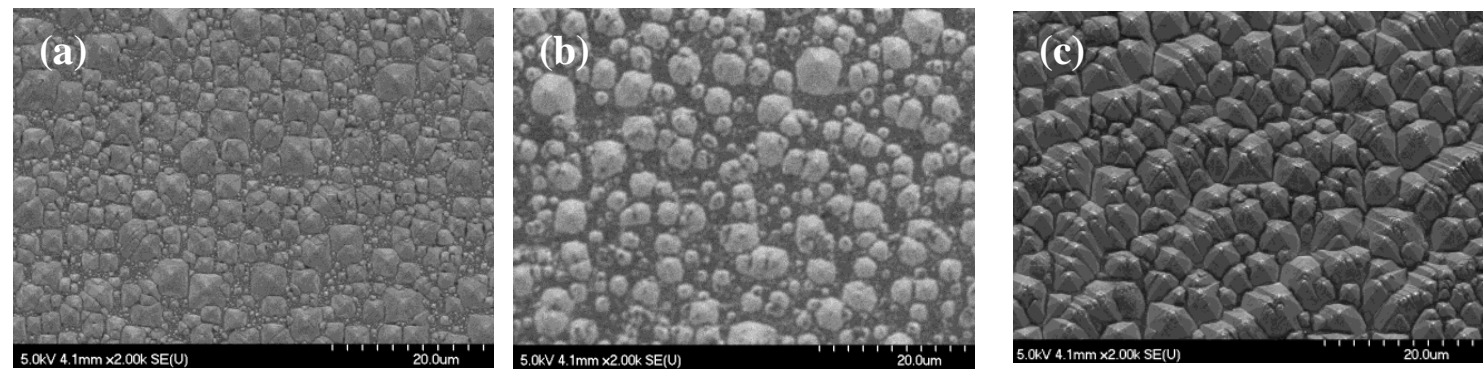

Figure 12: The SEM image of the (a) higher-velocity region of the non-optimized (non-circled region of figure 11b), (b) lower-velocity region of the non-optimized (circled regions of figure 11b) and (c) lower-velocity region of the optimized inlet (circled regions in figure 11a)

The efficacy of the new design in improving the reflectance of the lower-velocity regions is illustrated in figure 13. As it may be noted, the lower-velocity regions of the optimized inlet (figure $13 \mathrm{c}$ or figure $12 \mathrm{c}$ or circled regions in figure 11a) present practically the same low reflectivity as the one observed in the higher-velocity areas (figure 13a or figure 12a or noncircled region in figure $11 \mathrm{~b}$ ).

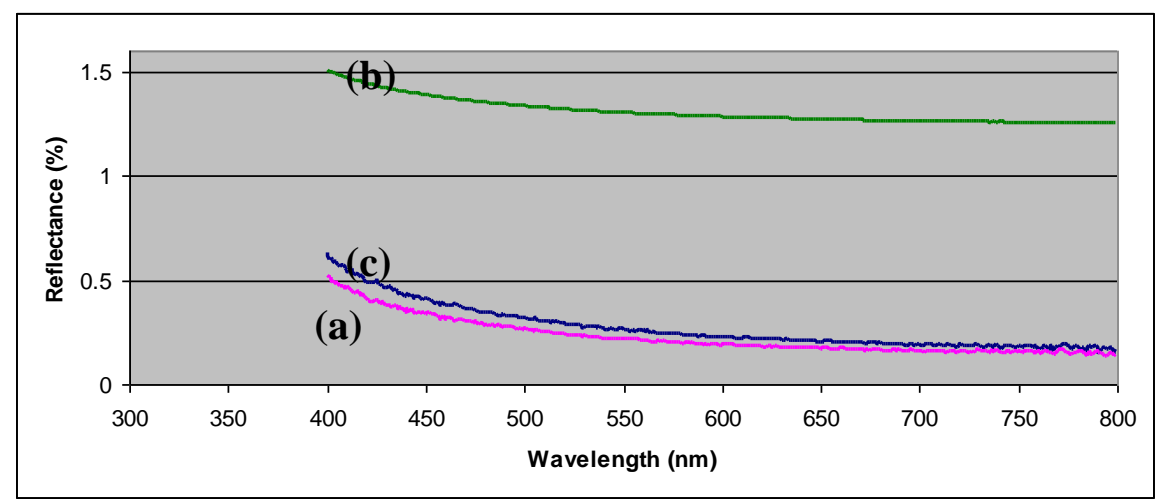

Figure 13: The reflectivity spectrum of (a) higher-velocity region of the non-optimized, (b) lowervelocity region of the non-optimized and (c) lower-velocity region of the optimized inlet

\section{Conclusions}

In this study, COMSOL tool was used to model and simulate the fluid flow patterns inside the GLE silicon texturing system. This allowed a better understanding of the influence of the fluid 
velocity on the texturing performance and the subsequent characteristics of the textured silicon in terms of morphology and surface reflectivity. The fluid velocity profiles obtained from the simulation tool suggested a good correlation between the location of high-reflectivity (and low surface coverage) areas with that of low fluid velocity regions. These regions are mostly located at the heights close to the inlet and at widths closer to the side walls as observed both experimentally as well as in the case of the simulation.

Based on the fluid velocity distribution predicted by the model and the obtained experimental results in terms of surface coverage and reflectance, it was concluded that the best performance of surface texturing requires some critical fluid velocity that seems to be beyond $0.047 \mathrm{~m} / \mathrm{s}$.

Various simulations were performed using different inlet geometry designs and the best case was found to be a slit of $1.2 \mathrm{~mm}$ gap all along the entire width of the inlet with rounded corners.

As a result of this new and improved inlet design, the low-velocity regions were drastically reduced resulting in a much more uniform reflectivity on the surface. In fact, with the new inlet design, the areas close to the inlet and the side walls (lower-velocity areas observed in the original inlet design) show morphology and surface reflectivity values very similar to those of the highervelocity areas as the fluid velocity reaches and goes beyond the critical $0.047 \mathrm{~m} / \mathrm{s}$ value.

Since the lower-velocity areas represented more than $15 \%$ of the total textured area in the case of the non optimal inlet design, the reduction and even elimination of these regions will be more critical in the scaled up process if the GLE approach is to be used as a viable alternative to the conventional texturing techniques at an industrial scale.

\section{References}

[1] Merlos A, Acero M, Bao M H, Bausells J and Esteve J 1993 TMAH/IPA anisotropic etching characteristics Sensors and Actuators A: Physical 37-38 737-743

[2] Amouzgar M, Kahrizi M 2012 A new approach for improving the silicon texturing process using gas-lift effect J. Phys. D: Appl. Phys. 45105102

[3] Comini G, del Giudice S 1985 A (k-epsilon) model of turbulent flow, Numerical Heat Transfer 8 133-147.

[4] Tanner F X, Zhu G-S and Reitz R D 2001 A turbulence Dissipation Correction to the kepsilon Model and Its Effect on Turbulence Length Scales in Engine Flows International Multidimensional Engine Modeling User's Group Meeting at the SAE Congress Detroit [5] Harlow F H, Nakayama P I 1968 Transport of turbulence energy decay rate Combust. Flame 30 45-319

[6] Jones W P, Launder B E 1972 The prediction of laminarization with a two-equation model of turbulence Int. J. Heat Mass Transfer 15 301-314

[7] Launder B E, Spalding D B 1974 The numerical computation of turbulent flows Comp. Methods in Appl. Mech. And Eng., 3 269-89

[8] Bardina J E, Huang P G, Coakley T J 1997 Turbulence Modeling Validation, Testing and Development NASA Technical Memorandum 110446

[9] Launder B E, Sharma B I 1974 Application of energy dissipation model of turbulence to the calculation of flow near a spinning disc Letters in Heat and Mass Transfer, 1-2 131-138 [10] Papet P, Nichiporuk O, Fave A, Kaminski A, Bazer-Bachi B, Lemiti M 2006 TMAH texturisation and etching of interdigitated back-contact solar cells Materials Science 241043 1049

[11] Merlos A, Acero M, Bao M H, Bausells J and Esteve J 1993 TMAH/IPA anisotropic etching characteristics Sensors and Actuators A: Physical 37-38 737-743 